\title{
Computer Generated Patient Plans Based on Patterns of Care
}

\author{
Ole Martin Winnem \\ NTNU, SINTEF, S.P. Andersensvei 15b, Trondheim, Norway \\ ole.M.Winnemasintef.no
}

\begin{abstract}
This article presents a method to create patient plans from Patterns of Care (PoC). PoC's are either developed based on Clinical Guidelines or as suggested in this article, based on generalized patient plans. The PoC's are then the basis for generating patient plans. The patient plan generation is a two step process where the first step is based on knowledge intensive case based reasoning and identifies the $\mathrm{PoC}$ to be used. The second step adapts the $\mathrm{PoC}$ to a patient plan. The way this approach uses PoC's and measured patient information to generate plans is a promising way of provide best practice and to reduce the work required by the medical experts.
\end{abstract}

\section{Introduction}

The medical community has so far been concentrating on developing Clinical Guidelines (CG) for manual use. Since these CG's has been developed for human interpretation and adaptation, they are not machine-interpretable. In order to create human supported automated processes, the CG's must be instantiated and structured. In our approach we introduce some context in the adaptation of the CG's in order to fit a specific guideline to a given care model. The care model we are using as example is based on a WHO care model [1,2] for treatment and care of chronic conditions, where patient plans are becoming more and more important.

In general patient plans are developed based on the experience of the medical expert and with no interaction with other professions. Reasons for this are the lack of tools to support a collaborative approach and the organisational model used. This article identifies components that are needed to improve the creation of patient plans and suggest a model that link the identified components together.

\section{The Clinical Guidelines - Structure and Content}

Clinical guidelines are normally presented in a text document with minimal structure. They have been developed in order to help physicians in performing their work in a unified way - following best practise, but as generic that adaptation to situation and care model is needed. Below is a figure of a typical Clinical Guideline. The figure shows a Clinical Guideline with chapters. These chapters are the only structure that is introduced and the content is based on descriptive text that is easy to understand for 
medical experts. This semi-structured representation is not possible for a computer to understand, but the second step that is performed by a knowledge engineer with medical domain knowledge which adapts it into a structured guideline represented in XML. Asbru is developed with focus on representing time constraints. This makes Asbru an alternative to the Creek [6] representation we are adapting. Creek is a semantic network representation with focus on representing general knowledge in combination with case specific information. We have chosen to use this because of its descriptive power and easy access to adapt it to our needs.

Common to these solutions are that they are only in limited use and that it costs a lot to build up them and to update and support them. Anyway we believe that it is an advantage to use case specific information and case based methods to identify the PoC to use, due to a simple and easy to understand way of updating the systems knowledge.

\section{Structure of PoC}

The PoC structure is defined in order to make it easy to include the information into a knowledge model and at the same time be expressive enough to capture all the vital information from a CG. In order to find this simplified structure we have studied CG's in order to identify generic structures. Generic structures alone are not enough and we have identified structures specific to diagnose and care model. Below is a part of a generic structure of the PoC. The structure shows how the PoC is built up, using XML. The most important part of this structure is the plan part that is used to create a specific plan for the patient. It contains information about all activities that should be created for the selected PoC. The activities have commonalities, but also differences. Typically, medication activities have information describing the ATC-Code and volume, which are parameters that are not useful for a nurse visit activity. In a nurse visit the human tasks describing what is needed to be performed and how this should be done following best practice is much more relevant.

The second important part is the structure that is matched to the patient diagnose and measurements. This part is used in order to select the PoC to be used for creating the patient plan. It is the major diagnostic parameters set by the medical expert. These are used in combination with the measurements that have been performed. Examples of diagnostic parameters are the ICPC or ICD10 code and parameters describing how sever the situation is. Examples of measured parameters are PEV or blood pressure used in the diagnostic process for a COPD or asthma patient. Further the structure contains a pointer to which CG it represents and eventually what level of the CG it is related to. It also includes general information about the purpose and care model it is related to.

The XML structure is used to build up a computer model that makes it possible to evaluate which PoC to use and how to adapt it into patient plans. The XML structure should be easy to understand both for human and computer, which makes it difficult to develop. The suggested structure will be further developed in order to fit new needs that are experienced during the test and evaluation period. 


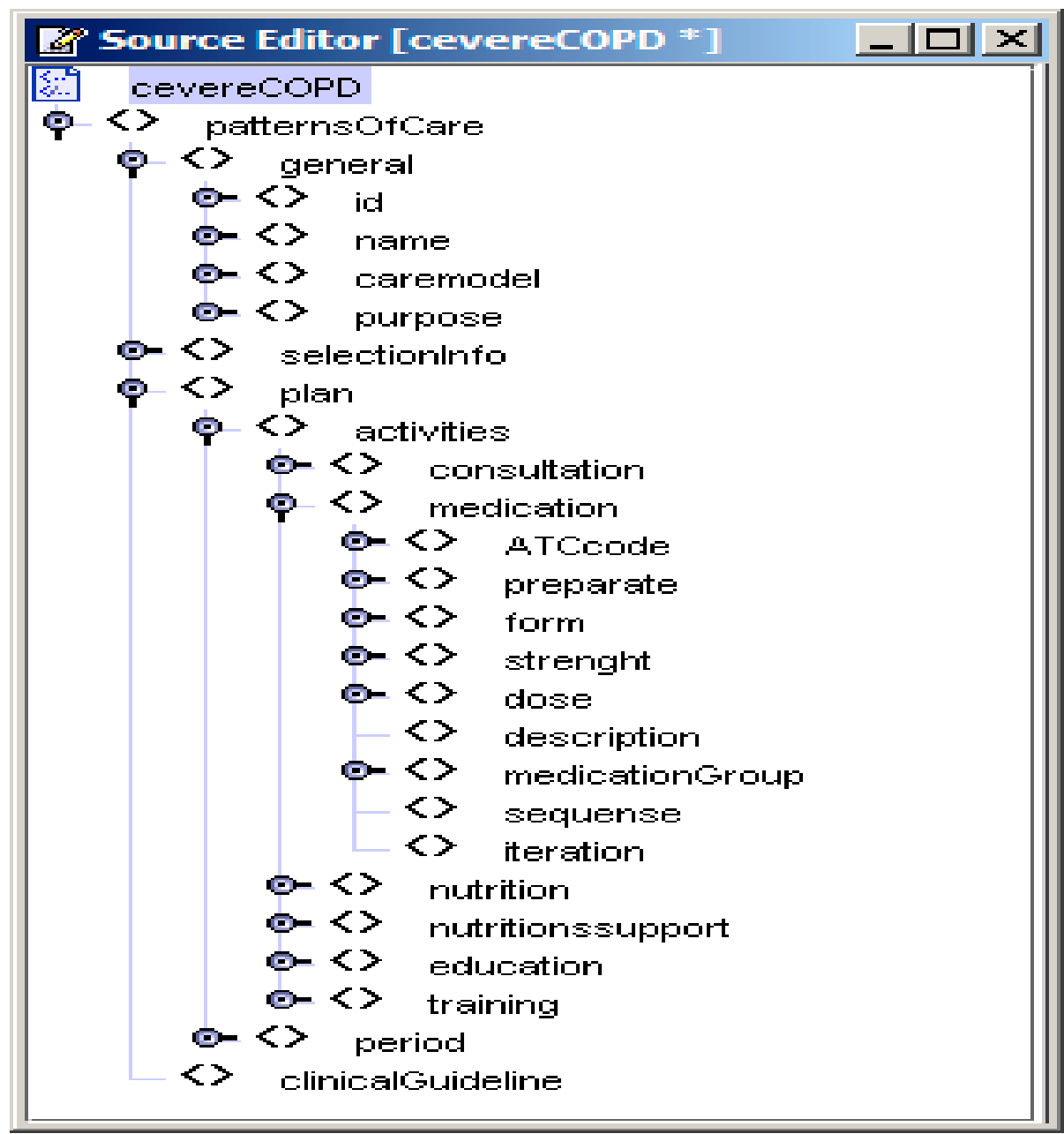

Fig. 2. An XML diagram of PoC

\section{Creating PoC}

At the moment the process of creating PoC from CG is based on a manual process with technology support. Our initial thoughts have been to design a semi-automated tool which gives knowledge support to the medical expert creating PoC based on CG. But because of the structure of CG's (created with textual structure to be easy to read for humans - not machines), we find it promising to start in the other end.

The similarity between $\mathrm{PoC}$ and a detailed patient plan makes it promising to study the possibility to create $\mathrm{PoC}$ from patient plans. This requires that the patient plans have been evaluated and controlled that they follow related clinical guideline. So far 
we have not gained experience with this method, but the results using this approach will be published as soon as the solution has been tested. The process suggested is a tree step process as follow:

1. Identify representative patient plans

2. Generate PoC from the identified patient plans

3. Evaluate and verify the resulting PoC according to related CG.

In the first step a team of experts identifies detailed plans that represent the diversity of the treatment for the selected CG. These plans are then categorized and made available for the induction algorithm that creates a generic plan or Patterns of Care. Before using this newly created $\mathrm{PoC}$, it should be evaluated up against related $\mathrm{CG}$. This is a manual process where the expert panel can edit the $\mathrm{PoC}$ in order to fit it to the CG.

\section{Knowledge Model and Resources}

The knowledge model in the system is based on a semantic network model describing all terms in the system and their relations to each other. This semantic network is combined with a case based retrieval system that uses the semantic model to explain the similarity between previous used plans and the new current problem to be solved. The knowledge representation system is an adapted and extended version of CREEK $[6,7]$. The explanation power is based on the product of all relations needed to explain how one term relates to another.

Other resources in the system are database resources related to plans for the care givers and patient constraints. The database resources are relational databases available to select consultation time with needed care providers. The most important care provider within advanced homecare is the home care unit [1]. This unit is the centre of the care provision for advanced homecare and it is the unit with the largest number of constraints. The needs of the patient are also most related to this resource unit. The patient constraints are related to medication, measurements, travel information, nutrition, patient knowledge level and available local caregivers (patient relatives), where the medication constraints are related to CAVE and mismatch between medications. Measurement constraints are related to problems regarding time of measurement and measurement dependability of medication.

\section{Generating Patient Plans}

The creation of patient plans from PoC contains two sequential steps. The first step is related to selection of $\mathrm{PoC}$ and the second is related to adapting the selected PoC to the available resources.

The selection of PoC is the most knowledge intensive and difficult task. Diagnose and available measurements give the input information which is mapped to available PoCs in order to select the most promising PoC. The selection of PoC is based on the CREEK [6, 7] retrieval engine which is able to match both quantitative and explain 
qualitative information and in this way it is much suited to match input information about the patient with the information represented in the PoCs.

Adapting the selected $\mathrm{PoC}$ to a patient plan is an optimisation problem which includes among others the constraints described in chapter 5. At the moment the optimisation in the system is only based on selecting the nearest resource and adding activity to the plan based on a sequential approach where the $\mathrm{PoC}$ is evaluated starting on the first activity.

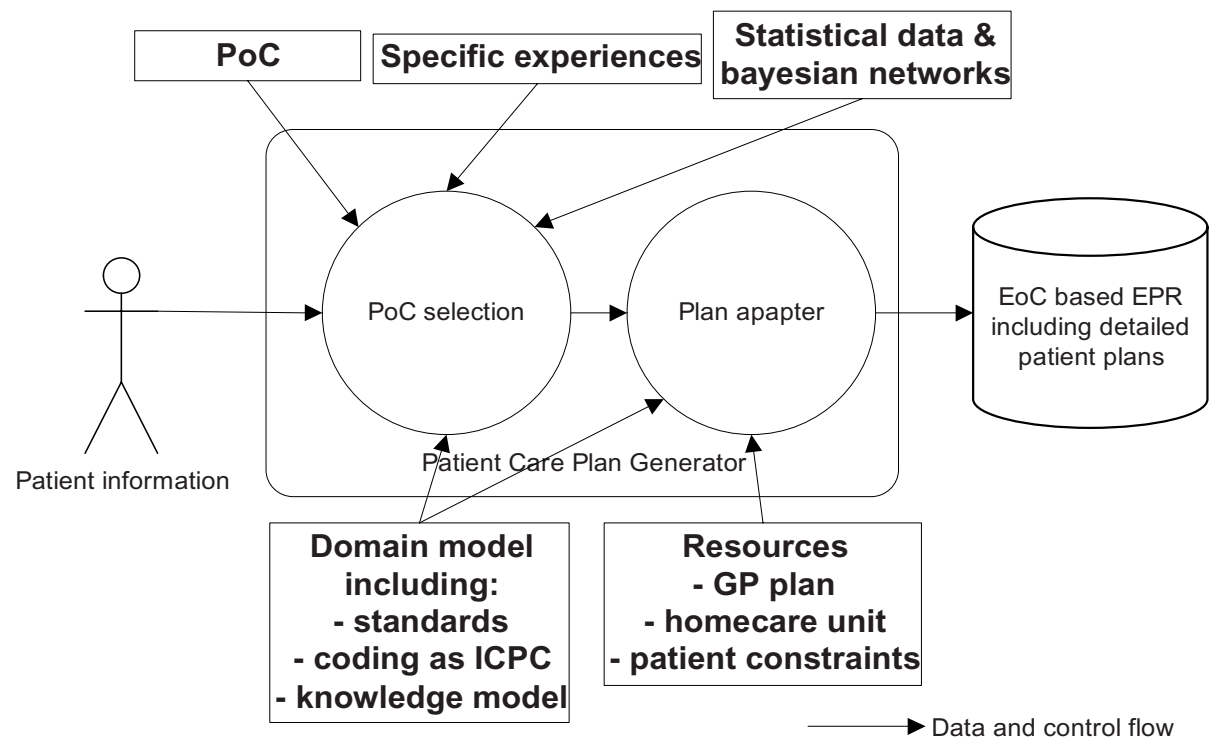

Fig. 3. The figure shows the two processes related to the Patient Care Plan Generator. The two processes uses different kind of information and knowledge sources in order to create patient plans

\section{Conclusions and Further Work}

This article has described a method for generating patient plans based on predefined Patterns of Care. This is an approach that will shorten the time used to develop plans for the treatment and care of chronic patients. Since the approach is based on structured clinical guidelines, it is reason to believe that the method will help giving the patient unified care according to best practice. In order to make this approach viable it is needed to have access to patient data and care giver resources. Access to these data sources are under development in Norway and the first projects where patient plans have been shared between caregivers at different level and profession have given promising results in 2003 . 
The design has not been tested in a laboratory or real world environment. This should be done in order to make further conclusions. The system will be further developed in order to make it possible to test the real use of $\mathrm{PoC}$ as standard for patient plan generation. At the end a large scale trial should be performed in order to make the final conclusions.

\section{References}

1. Winnem, O.M., Walderhaug, S. Distributed, role based, guideline based decision support. in eHealth in Common Europe. 2002. Krakow: Springer.

2. WHO, Innovative care for chronic conditions. 2002, World Health Organization: Geneva. p. 61.

3. van Bemmel, J.H., Musen, M.A., Handbook of Medical Informatics. 1997: Springer. 621.

4. Shahar, Y., Miksch, S. \& Johnson, P., The Asgaard Project: A Task-Specific Framework for the Application and Critiquing of Time-Oriented Clinical Guidelines. Artificial Intelligence in Medicine, 1998. 14: p. 29-51.

5. Shahar, Y., Young, O., Shalom, E., Moskovitch, D., Boaz, D. and Galperin, M. A Hybrid Framework for Representation and use of Clnical Guidelines. in AMIA. 2002.

6. Winnem, O.M., Integrating knowledge- and symbol level modelling - THE CREEST WORKBENCH, in IDI. 1996, NTNU: Trondheim. p. 134.

7. Aamodt, A.P., E, Case-Based Reasoning: Foundational Issues, Methodological Variations and System Approaches. AICom - Artificial Intelligence Communications. Vol. 7:1. 1994: IOS Press. 39-59. 